Accepted for publication in "International Psychogeriatrics"

Note: This is an uncorrected version of an author's manuscript accepted for publication. Copyediting, typesetting, and review of the resulting proofs will be undertaken on this manuscript before final publication. During production and prepress, errors may be discovered that could affect the content.

\title{
It is not only memory: Effects of SenseCam on improving well-being in patients with Mild Alzheimer Disease
}

Ana Rita Silva

Faculty of Psychology and Educational Sciences, University of Coimbra, PORTUGAL

\section{Maria Salomé Pinho}

Faculty of Psychology and Educational Sciences, University of Coimbra, PORTUGAL

\section{Luís Macedo}

Department of Informatics Engineering, University of Coimbra, PORTUGAL

\section{Christopher Moulin}

Laboratories de Psychologie \& NeuroCognition (CNRS UMR 5105), Université

Grenoble Alpes, FRANCE

\section{Maria Salomé Caldeira}

Coimbra University Hospitals, Coimbra, PORTUGAL

\section{Horácio Firmino}

Coimbra University Hospitals, Coimbra, PORTUGAL

Corresponding author:

Ana Rita Silva

Faculty of Psychology and Educational Sciences of the University of Coimbra

CINEICC - Cognitive and Behavioural Center for Research and Intervention 
Colégio Novo Street, s/n

3001-802 Coimbra

PORTUGAL

$+351239851450$

ana.rita.ess@gmail.com

\begin{abstract}
Background. Cognitive interventions (either restorative or compensatory) developed for mild Alzheimer's Disease (AD) have been tested widely with cognitive measures, but less is known about how the effects of such interventions are generalizable to daily functioning. In the present study, we looked at affective state and perceived functionality and quality of life indicators, for three different cognitive rehabilitation programmes.

Methods. Fifty-one AD patients in the mild stage of the disease were selected for the study and were randomly assigned to one of three cognitive training groups: 1) Memo+ (a paper and pencil memory training program); 2) SenseCam (wearable camera used as a passive external memory aid); 3) Written diary (a personal journal, used as control condition). All patients attended eleven sessions, twice a week, of one-hour length. The three outcome indicators were examined with standardized instruments applied before the intervention, one week after and at six months follow up.

Results. After treatment, the SenseCam and Memo+ groups had significantly reduced depressive symptoms compared to the Diary control condition. The same was found for measures of perceived functional capacity. No intervention effects were found for quality of life measures. The immediate effects of the interventions were not maintained at follow-up.

Conclusions: Our results suggest that two types of memory rehabilitation can improve depressive symptomology and instrumental activities of daily living, suggesting that these interventions can stimulate not only cognition but also wellbeing, at least in the short term.
\end{abstract}

Keywords: Alzheimer's disease, SenseCam, Depression, Quality of life, Functional capacity. 
Introduction

Alzheimer's disease (AD) represents a major challenge for healthcare as it includes a set of symptoms that contribute to poor perceived quality of life for these patients. The progressive deterioration of memory function is one of the core symptoms of this disease and one of the most disruptive in the mild stages (Jahn, 2013). In particular, deficits in autobiographical memory (memory for personally experienced events/general information related to the maintenance of the self) contribute significantly to this disruption, due to their close relationship with self and identity. For a recent review of the link between self, identity and memory see Prebble, Addis and Tippett (2013).

It is known that self concepts and identity, as stored in autobiographical memory, are related to wellbeing in older adults (Rathbone, Holmes, Murphy and Ellis, 2015). In Alzheimer's disease particularly, the progressive decrease of memories that support the person's self concept influences patients' well-being (e.g. Seidl et al., 2011). As such, memory deficits are often associated with non-cognitive symptoms frequently identified by patients with $\mathrm{AD}$ and their carers, such as anxiety, depressive mood, apathy, and decline in functional abilities. Such symptoms tend to appear progressively beginning in the "pre-clinical" phase of the disease (Baquero and Martin, 2015). Depressive symptoms are actually the most common affective symptoms experienced by $\mathrm{AD}$ patients in the Mild stages of the disease. This combination of cognitive, affective and functional impairment often leads to what has been described as a burden for both the patients and their caregivers, caused by the "excess disability" of the AD patient (Bahar-Fuchs et al., 2013).

Reviews of pharmacological and non-pharmacological interventions that delay or compensate for the clinical decline of patients with AD routinely consider non-cognitive symptoms as important as cognitive deficits(Cammisuli, Danti, Bosinelli and Cipriani, 2016; Olazarán et al., Salomone, Caraci, Leggio, Fedotova and Drago, 2012; Zec and Burkett, 2008). However, the focus has tended to be on cognitive symptoms and the cognitive effects of interventions (e.g. Bahar-Fuchs et al., 2013), even if researchers and clinicians consider that interventions are only effective if they target the full spectrum of symptoms (e.g. Huckans et al., 2013). In this article, we turn our attention to the effects of cognitive rehabilitation of patients in AD to measures of well-being and mood. An 
important distinction in cognitive rehabilitation is the difference between internal aids (such as training or strategies to improve memory) and externals aids which are devices or materials which exist in the environment (e.g. Piras, Borella, Incoccia and Carlesimo, 2011).

Internal interventions, which include cognitive rehabilitation, aim to help AD patients at the beginning of the disease to manage its impact. They consisted, at first, of intensive sets of memory training sessions, aimed at stimulating specific cognitive functions. However, these first programmes were somewhat ineffective due to the substantial cognitive effort they required of the patients, particularly considering the cognitive abilities already impaired at that point of the disease (see Kapur et al. 2004 for an overview). The difficulty of such demanding tasks and the subsequent failures perceived by the patients contributed to dropout, feelings of frustration and a decrease of motivation, both for the patients and their relatives and carers (Logsdon et al., 2007). Some techniques, such as errorless learning (Hampstead et al., 2012), spaced retrieval (Lee et $a l ., 2009$ ) and using vanishing cues (Haslam et al., 2011) are less demanding and more helpful for patients' memory functioning (Clare et al., 2010). Such strategies are judged as being able to stimulate specific cognitive abilities in patients with cognitive impairment (e.g., Arkin, 2001; Cherry et al., 2010; Choi and Twamley, 2013; Dun and Clare, 2007; Fish et al., 2010).

The challenge with such rehabilitation programmes is to transfer the improvements from laboratory based tasks and materials to real life situations; and in general the effects were circumscribed to specific measures and not generalized to daily functioning (Bahar-Fuchs et al., 2013; Bellevile, 2008; Martin, Clare, Altgasse, Cameron and Zehnder, 2011). Nonetheless, cognitive rehabilitation has had impacts on activities of daily living and well-being when the rehabilitation has focussed on daily life tasks, such as re-learning how to use voice-mail, how to knit, or the names of members at a social club (e.g. Thivierge et al., 2008). As such, intensive, and person-centred rehabilitation has been seen to influence patient well-being, but most of the work in this area has been reports of successful case-studies.

Despite this growing evidence towards the most recent cognitive enhancement techniques based in the practice of internal strategies for learning and remembering, most of the 
recent data has suggested that external memory aids, which are less demanding for the person for their use to be effective, are more pertinent for patients with degenerative diseases (Piras, Borella, Incoccia and Carlesimo, 2011). In fact, external memory aids are based on the premise that one must, in an irreversible (so far) neurodegenerative disease, help patients to compensate for lost cognitive abilities. Consequently, aids such as diaries, journals, calendars, and wearable cameras, have been used in daily life, and here there has been a more direct influence on patients' daily life. The close link to everyday function of these aids compared to other cognitive interventions make them a more suitable means of cognitive enhancement in AD (Huckans et al., 2013). Particularly, the fact that some external aids do not require training to be used - so called "passive" cognitive aids - is less burdensome for patients, carers and healthcare professionals.

Due to several technological advances at the beginning of this century, wearable cameras such as SenseCam (Berry et al., 2007) appeared in the field of cognitive rehabilitation for people with brain injury. These devices are not cognitively demanding for patients, since they are just required to wear the camera and then passively review the collected images later. A review of the early work in using such devices to improve memory function, suggests that such devices mimic autobiographical memory function, and deliver measureable effects on memory performance, both for the contents of the events depicted in the images, and for memory function more generally (see Silva et al., 2016, for a review of the link between SenseCam and memory function).

The fact that SenseCam targets autobiographical memory function (and enhances the specificity of the memories retrieved) is a critical issue when considering transfer. It is expected that cognitive training with SenseCam could reduce depressive symptomatology (Gonçalves, Albuquerque and Paul, 2009). This reasoning is largely based on the observation that training memory specificity in depressed people leads to changes in depressive symptomology (decreases in rumination and experiential avoidance, and increases in problem-solving skills; Raes, Williams and Hermans, 2009). Moreover, the experience of using SenseCam has been described by patients with several neurological conditions (e.g. temporal limbic encephalitis, Berry et al., 2007) as a pleasant experience, increasing self-confidence in memory function as well as reducing anxiety (Hodges et al., 2006; Berry et al., 2007; Loveday and Conway, 2011). These findings were also corroborated in De Leo et al.'s (2011) study, that adapted the paradigm of wearable 
cameras to a smart-phone and tested its effects in one AD patient. However, most of this data was collected by asking the patients and their carers about what they remembered and how they felt, without structured and standardized measures.

So far, from the studies that tested SenseCam as an external memory aid, only one study with healthy older adults tested subjective feelings about using SenseCam with objective measures (Silva et al., 2013). In this study, subjective measures (ratings in a Likert scale from one to seven) were taken for the following variables: 1) vividness of events; 2) the ability of the memory aid's to prompt additional memories; 3) surprise; 4) excitement; 5) feeling of alertness; 6) value of the memory aid; 7) feeling of re-experiencing the events. The measures from these variables were in line with the effects on cognition as tested by neuropsychological assessment instruments. As such, when the scores in standardized tests of memory, executive function, attention, increased, the ratings on the subjective variables increased.

Until now, only three studies using SenseCam as a memory aid examined people with Alzheimer disease (Lee and Dey, 2008; Crete-Nishiatha et al., 2012; Woodberry et al., 2015). Of these studies, only Woodberry et al. (2015) tested the efficacy of SenseCam with a group of patients. They measured the ability to remember events depicted in the SenseCam images, but also their subjective experience. They found that the patients who benefited most from the camera by remembering more events/details, were those who rated the experience of using the camera as more pleasant and helpful.

In sum, there is a need to consider and measure the impact of cognitive rehabilitation methods on quality of life, mood and the capacity to carry out daily activities, an emphasis which is clear in the pharmacological intervention literature. In comparison, the impact of cognitive rehabilitation on these critical entities is less well known. Critically, different types of rehabilitation may have different effects on well-being and mood. First, intensive memory training, whilst improving specific cognitive function, may not transfer to other activities, and may be perceived as a difficult, and time-consuming activity. It may also upset patients by constantly challenging and testing their memory function. Second, more active external aids, such as keeping a journal, may also not lead to any real transfer, and again, they may be experienced as effortful, unenjoyable or not beneficial, although we would not expect them to upset or frustrate the patient in the same way as memory 
training. On the other hand, keeping a journal about recent events may support and maintain autobiographical memory function in a way memory training does not, and therefore it may have some positive effects on identity. Finally, passive external aids such as SenseCam, may offer some stimulation of memory without challenging or upsetting the user, and in turn, this method will also support autobiographical memory, and therefore the identity and well-being of the patient. We tested the impact of these three types of memory rehabilitation on various measures of well-being.

We report here the data pertaining to wellbeing from a research programme which has shown that in comparison to a journal control condition, SenseCam and a comprehensive memory training program named Memo+ (see method section for description) improve episodic, semantic and working memory (Silva et al., submitted). These benefits were maintained at a six-month follow-up. SenseCam was particularly effective in improving autobiographical memory, compared with the other two interventions, which as we have evoked above, might lead to an increase in well-being through the maintenance of the self-concept. We might expect that any improvement in cognitive function would lead to improvements in the quality of life and well-being, since the literature suggests that the measures of non-cognitive variables such as mood, functionality, perceived quality of life are associated with the effects of cognitive interventions in cognition. This means that we hypothesised that alongside the cognitive benefits of SenseCam and Memo+ we should see positive changes in non-cognitive measures of wellbeing, mood and quality of life. However, the study is somewhat exploratory, and although there is plenty of evidence to suggest that memory and cognition changes drive changes in depressive symptomology, we could also reasonably expect that the SenseCam group will have more of a positive impact on mood and well-being, since keeping a journal and the Memo+ sessions may be seen as laborious and remind patients of their difficulties.

\section{Methods:}

\section{Design}

This study was a pre-test/post-test/follow up controlled trial of three cognitive training interventions. This study was single-blind randomized trial; the participants were unaware of the nature (control/experimental) of the intervention they were taking part in. 
Participants were randomly allocated to receive either Memo+ training, SenseCam training or to complete a daily journal (see the Cognitive interventions section for description of the groups). Seventeen participants were randomly assigned to each group. Neuropsychological assessment of both cognitive and non-cognitive measures was performed at baseline, one-week after the end of the interventions and at six-month follow up. For each group, the number of sessions was the same (eleven sessions, twice a week and one hour per session). All fifty-one participants took part in pre and post assessment sessions but only forty-six patients were in the follow-up visit (dropouts were due to the following reasons: two participants died during the follow-up interval and three participants changed address, missing the third visit). The therapist that conducted all sessions of cognitive training for all participants in all groups was the first author of this paper.

The study procedures were approved by the research ethics board of the hospital (CHUC ethics approval number 4212) and by the direction of the remaining recruitment institutions, and we obtained written informed consent from all participants prior to the start of the study. No financial compensation was provided to the patients for their participation in the study.

Cognitive interventions

SenseCam Group. This group's cognitive training activities consisted of using SenseCam worn around the neck while freely performing routine activities, and the review, on a computer screen, with the neuropsychologist, the set of images captured by the camera. SenseCam was used to capture images from participants' daily life for the whole period of the intervention (six weeks). The review sessions occurred twice a week (with a faceto-face meeting with the neuropsychologist, as for the other conditions). In the three-day interval between each session, participants wore the camera every day for the longest possible time in order to maximize the number of images gathered. Participants were additionally informed that they could remove or turn off the camera if they wanted any moment/event to remain private. All the images gathered in those three-days were reviewed during the following session. Approximately 7000 images were shown to the participants on a computer. This sequence was repeated for the eleven sessions in the intervention. Besides being asked to comment on the pictures they were watching, during 
the session participants did not receive any feedback from the therapist about the comments' accuracy. Patients and their caregivers were instructed, before starting to use the device, on how to charge it and how to use the buttons (privacy, on/off button, manual trigger).

Memo+ Group. We constructed a cognitive training program based on pencil and paper tasks to practice motivation, attention and memory, with progressive levels of difficulty throughout the sessions. We selected tasks and techniques where there was published evidence of memory improvement in brain injury and we included training and practice in the following domains: exercises to improve motivation, attention, working memory, autobiographical and episodic memory, semantic memory and implicit memory. An adapted motivational interview was constructed for the start of the sessions (including a set of open questions concerning the interest of the participants in taking part in the study, expectations about possible gains to be obtained after the study, and also questions regarding the prior existence of self-initiated activities to stimulate the brain - such as crosswords, Sudoku, reading, etc.). Some psychoeducation was given at the first session about neurodegenerative diseases and the content of the cognitive training sessions. These sessions involved the practice of cognitive strategies for learning and retention of information (spaced retrieval, vanishing cues, errorless learning, mnemonics). Exercises had progressive levels of difficulty throughout the training sessions. Each session featured two exercises of explicit memory (e.g. see a photograph of a person's face together with her/his name and create a mnemonic that links the face to the name, ex: Maria has brown curly hair, then recall the names when only the faces are presented and then recall them using the spaced retrieval technique, e.g., recall after one minute, three minutes, five minutes to a maximum 12-minute interval) and one exercise of implicit memory (e.g. read a set of instructions and perform the actions depicted in those instructions, e.g., make a call to someone from a list of contacts). At the end of each session, feedback from the neuropsychologist was given concerning the performance of the participant on each exercise.

Personal Diary Group (Control Condition). In this intervention group, participants were asked to write down their daily activities in a personal journal with sections to fill in and to then read to the experimenter in each session. The diary was organized into sections on a one-page-per-day basis. Sections were the following: Event description (where the 
participant writes the activity done that day, for example, "I had lunch with my husband"); Time (where the participant had to register the time of the day the event took place, for example, "12.30 p.m."); place (to fill with information about where the event took place, for example, "in my dining room"); people involved (where the participant had to describe other people that were part of the event described, for example, "my husband Luis"); and emotional description (where the participant was asked to described how they felt during the event, for example, "happy; hungry"). Two other sections were also included as aids for prospective memory: Appointments and Other Notes. Participants were instructed to fill the diary pages at the end of each day during the six weeks of the intervention, and carers were instructed to recall the participants about the need to fill the diary pages in case they don't perform that action by their own.

Non-cognitive outcome measures

\section{Depressive symptoms}

We used the Geriatric Depression Scale - 30 to examine participants' mood (GDS-30). This is a 30 -item scale with a yes/no response format, that examines the presence of psychological and behavioural symptoms of depression, during the last week. It is specifically developed for the geriatric population, excluding symptoms related with other somatic diseases. The final score is the sum of symptoms. The scale allows us to distinguish the absence of depressive symptoms (score between 0-10 points), mild to moderate depressive symptoms (score between 11-20 points) and severe depressive symptoms (score between 21-30 points)).

\section{Functional Status}

We assessed the perception of participants to perform routine activities of daily living, using the Adults and Older Adults Functional Assessment Inventory (IAFAI), a Portuguese inventory of functional incapacity assessment, specifically constructed for adults and older adults. The IAFAI includes both basic activities of daily living (BADL) and instrumental activities of daily living (IADL) to enable a comprehensive assessment of functional capacity. The 18 BADL items encompass four domains (feeding; dressing; bathing and continence; and mobility and transference). The IADL-familiar items encompass four domains (conversation and telephone use; meal preparation; housekeeping; and home security), and the IADL-advanced items encompass five 
domains (comprehension and communication; health-related decision making; finances, going out and transportation use; and leisure and interpersonal relationships). The inventory is administered in a structured interview format in order to determine for each item the functional capacity level.

Quality of Life

Quality of life is one of the measures more frequently tested when examining noncognitive outcomes of cognitive training. As such, we applied to all participants the European Portuguese World Health Organization Quality of Life-OLD (WHOQOLOLD) module, which consists of 28 items rated on a five-point scale covering 7 domains: Sensory functioning; Autonomy; Past, present and future activities; Social participation; Death and dying; Intimacy; and Family/family life (a domain that is only present in the Portuguese version of this module). Higher scores indicate better QoL.

\section{Participants}

Inclusion criteria for the study were the following: diagnosis of probable $\mathrm{AD}$, amnesic presentation (NIA-AA workgroup criteria, McKhann, et al., 2011); less than six-months since the diagnosis assignment; age range between 60 and 80 years-old; at least one year of formal education; score between 15 and 26 on the Mini-Mental State Examination (MMSE, Folstein, Folstein and McHugh, 1975); very mild (0.5) to mild (1.0) rates in terms of disease severity (Clinical Dementia Rating, CDR, Morris et al., 1993) absence of severe symptoms of depression (Geriatric Depression Scale, GDS, Yesavage et al., 1983); community-dwelling or integrated in a day care centre; and cared by an identified caregiver willing to participate in the study. Table 1 describes the demographic characteristics of participants at baseline in the three cognitive intervention groups. We used the Addenbrookes Cognitive Examination - Revised (ACE-R; Mioshi, Dawson, Mitchell, Arnold and Hodges, 2006) test to screen in more detail general cognitive function. We also used the Portuguese version of the National Adult Reading Test NART, (Teste de Leitura de Palavras Irregulares - TELPI, Alves, Simões and Martins, 2012) to examine the estimated premorbid IQ of the participants in the study.

Sixty-seven patients with mild AD volunteered to participate in the study, but only fiftyone completed the study; that is, 16 participants dropped before the study started, all for 
practical reasons. These participants were not even randomised into the study and did not start any of the rehabiliaition programmes. To compare these dropouts with the full sample, ANOVAs were carried out on the baseline and screening measures (no other measures were aviable) bwteen four groups: the three experiemental conditions and the 16 drop-outs. No differences were found for age $F(3,63)=2.23, \mathrm{p}=.10$ or for the screaning measures - ACE-R $F(3,63)=.473, \mathrm{p}=.70 ;$ MMSE $F(3,63)=.682, \mathrm{p}=.567 ;$ CDR $F(3,63)=.742, \mathrm{p}=.531$, with only differences found for the level of education, $F(3,63)=3.16, \mathrm{p}=.03$, where the dropouts differed significantly from the diary group participants, having less years of education (Tukey $\mathrm{LSD}=.01$ )) The age range of the particpants was 62 to 80 years, $M=73.65, S D=5.498$ ).

For the sample of 51 participants, One-way ANOVAs again demonstrated the absence of differences between groups for age, $F(2,48)=1.475, \mathrm{p}=.241$, level of education (years of formal education, $F(2,48)=2.340, \mathrm{p}=.109$, and estimated premorbid IQ (TELPI) $F(2,48)=.540, \mathrm{p}=.587$. The groups also did not differ on general cognitive status according to the ACE-R, $\mathrm{F}(2,48)=.406, \mathrm{p}=.669$, at baseline. The GDS scores are discussed in the results section. Recruitment and cognitive training took place both at the psychiatric and neurology services of Coimbra University Hospitals (CHUC) and at the Portuguese Alzheimer Disease Association (APFADA), between January 2013 and October 2015.

\section{INSERT TABLE 1 HERE}

Statistics

Analyses were conducted using the Statistical Package for Social Sciences 22.0 (SPSS 22.0; IBM SPSS, Chicago, IL). Data were examined and no outliers were identified. The distributions of data were examined using histograms, plots, and tests of skewness and kurtosis. All demographic characteristics and outcome measures followed normal distributions. Differences in the demographic characteristics between the groups were analysed using one-way ANOVAs. We also performed one-way ANOVAs to examine the differences between groups in the outcome measures at baseline, and the impact of cognitive training in those measures was analysed using $3 \times 3$ mixed design ANOVAs (with Group as the between subject factor and Visit as the within subject variable). 
Significant main effects and interactions were analysed using pairwise comparisons, with Bonferroni's adjustment for multiple comparisons. The $\alpha$ value was set at .05 for all statistical tests, and at .01 for interactions. Differences in each training group performance in the outcome measures between baseline (Visit 1), immediately after training (Visit 2) and six months' follow-up (Visit 3) were analysed using paired samples t-tests. Setting the $\alpha$ for the study involved consideration of the fact that multiple comparisons were used on the same data in this study, which would generally lead to using a more conservative $\alpha$ to avoid Type I errors. However, the study also involved exploration of a new cognitive training interventions with a relatively small clinical sample. This meant that it was equally important to avoid Type II errors, possibly missing information about the potential effectiveness of the program. Therefore, no statistical adjustment was made for multiple comparisons.

Results

We first compared the group scores at baseline for outcome measures outlined above. One-way ANOVAs showed no significant pre-intervention differences between groups on any of the measures: Geriatric Depression Scale, $F(2,48)=.295, p=.342, \eta^{2}=.024$, Adults and Older Adults Functional Assessment Inventory, $F(2,48)=1.68, p=.145, \eta^{2}=$ .076. The differences between groups at baseline for the World Health Organization Quality of Life - OLD measure approached significance, $F(2,48)=2.62, p=.09, \eta^{2}=.079$ (Table 2, for means and standard deviations). We will now describe the results in the noncognitive outcome measures applied to participants in this study concerning the effects of the three cognitive training interventions.

\section{INSERT TABLE 2 HERE}

Depressive symptoms

A 3 x 3 mixed ANOVA was performed, with the cognitive training group as the between subjects' factor, in a total sample of 46 patients. A moderate main effect of Group was found, $F(2,43)=3.53, p=.03, \eta^{2} \mathrm{p}=.14$. Fisher LSD post-hoc comparisons revealed that 
depressive symptoms as assessed by GDS were lower for the SenseCam group in comparison to the Diary group $(p=.03)$, but no differences were found between the SenseCam and the Memo+ group ( $p=.51)$ and between the Memo+ Group and the Diary group ( $p=.26$ ). A moderate main effect was also found for Visit, $F(2,43)=23.39, p<.01$,

$\eta^{2} \mathrm{p}=.36$. LSD pairwise comparisons with Bonferroni adjustment identified lower scores in GDS in Visit 2 compared to both Visit $1(p<.01)$ and Visit $3(p<.01)$, and lower scores in Visit 3 compared to Visit $1(p<.001)$, with an effect of the intervention in decreasing the depressive symptoms. Finally, a strong interaction was found for Group x Visit, $F(2,43)=23.13, p<.01, \eta^{2} \mathrm{p}=.52$. Graph 1 shows evidence for this interaction and we analysed differences within groups between visits by performing paired samples t tests.

Considering each group separately, the SenseCam group showed a decrease of GDS scores at Visit $2(\mathrm{M}=6.79, \mathrm{SD}=3.66)$ compared to Visit $1(\mathrm{M}=12.64, \mathrm{SD}=6.24, t(14)=7.93$, $p<.01, d=1.24)$ with a strong effect size, but these increased significantly again at Visit 3 $(\mathrm{M}=7.57, \mathrm{SD}=4.03, t(14)=-2.47, p=.03, d=1.105)$ although the mean at Visit 3 is also significantly inferior than Visit 1 scores, $t(14)=5.17, p<.01, \mathrm{~d}=.965$. The Memo+ group depressive symptoms scores revealed a significant decrease (which was moderate in terms of strength) at Visit $2(\mathrm{M}=10.31, \mathrm{SD}=5.12)$ compared to Visit $1 \quad(\mathrm{M}=11.44$ $\mathrm{SD}=4.62,(t(16)=2.21, p=.04, d=.363)$, but showed only marginal differences between Visit 2 and $3(\mathrm{M}=11.06, \mathrm{SD}=4.50), t(15)=-1.91, p=.08, d=.218$, and no significant difference between visits 1 and 3, $t(15)=.67, p=.51, d=.137$. The Diary group GDS scores did not change significantly after the intervention, with no differences found between Visit $1(\mathrm{M}=13.00, \mathrm{SD}=5.29)$ and Visit $2(\mathrm{M}=13.40, \mathrm{SD}=5.22), t(16)=-1.93, p=.07, d=.076$, and there was even an moderate increase in the GDS scores at Visit $3(M=14.60$, $\mathrm{SD}=5.12)$, compared to both Visit $2, t(15)=-4.05, p<.01, d=.243$, and Visit $1, t(15)=.4 .58$, $p<.01, d=.328$.

\section{INSERT GRAPH 1 ABOUT HERE}

\section{Functional capacity}

The Adults and Older Adults Functional Assessment Inventory (IAFAI) yields a global score as well as scores for basic activities of daily living (ADL), instrumental-familiar ADL and instrumental-advanced ADL. We analysed as a main outcome the global score. A 3x3 Mixed ANOVA found no main effect for Group, $F(2,43)=.29, p=.791$. A main 
effect of Visit was found, $F(2,43)=16.26, p<.01, \eta^{2}{ }_{p}=.28$, and LSD pairwise comparisons with Bonferroni adjustment showed that participants rated their level of incapacity lower at Visit 2 compared to Visit $1(p<.01)$ but this returned to baseline rates at Visit $3(p<.01)$, with no differences found between Visit 3 and Visit 1 ( $p=.34)$. An interaction of Group and Visit was found, $F(2,43)=8.71, p<.01, \eta^{2} \mathrm{p}=.29$. It was found that for the SenseCam group, the level of incapacity perceived by participants decreased after the intervention at Visit $2(\mathrm{M}=11.51, \mathrm{SD}=7.52)$ relative to Visit $1(\mathrm{M}=20.55, \mathrm{SD}=8.64, t(16)=7.75, p<.01$, $d=1.40)$ but it increased again moderately at follow up - Visit $3(\mathrm{M}=16.92, \mathrm{SD}=8.36)$, compared to Visit $2, t(14)=-3.58, p=.01, d=.777$, returning to ratings closer to baseline Visit $1, \mathrm{t}(14)=1.64, p=.13, d=.479$. The same pattern was found in the Memo+ group, were the ratings of incapacity decreased strongly at Visit $2(\mathrm{M}=13.63, \mathrm{SD}=7.42)$ compared to Visit $1(\mathrm{M}=21.29, \mathrm{SD}=11.19), t(16)=5.23, p<.01, d=.808)$, but returned closer to the Visit 1 ratings level at Visit 3, $\mathrm{M}=18.26, \mathrm{SD}=9.62, t(14)=1.72, p=.10$, $d=.281$ ). The Diary group scores followed a different pattern to the other groups, with the ratings of perceived incapacity increasing moderately after the intervention, at Visit 2 $(\mathrm{M}=18.89, \mathrm{SD}=7.12)$ compared to Visit $1(\mathrm{M}=16.17, \mathrm{SD}=5.63), \mathrm{t}(16)=-3.08, p>.01$, $d=.424)$. They maintained stable at follow-up, Visit $3(\mathrm{M}=19.59, \mathrm{SD}=-7.33)$ not being different to Visit 2, $\mathrm{t}(14)=-.76, p=.34, d=.097$ but increased scores compared to Visit 1 , with a moderate difference $\mathrm{t}(14)=-2.71, p=.02, d=.53$.

The IAFAI also provides partial scores for the basic ADL, the instrumental familiar ADL and the instrumental advanced ADL. As referred to in the literature (Sikkes et al., 2011), mild AD functional impairments are mainly related to instrumental ADL, mostly the more complex/advanced activities, whereas more severe stages of the disease show a progressive compromise of basic ADL. We analysed the ratings of the two instrumental ADLs. We analysed the ratings of incapacity for the Familiar IADL and the Advanced IADL. For the first score, no main effect for Group was found, $F(2,43)=.12, p=.89$, $\eta 2 p$ $=.014$. However, there was a main effect for Visit, $F(2,43)=5.31, p<.01$, an effect described as relatively weak, $\eta 2 p=.11$. LSD pairwise comparisons with Bonferroni adjustment revealed than in Visit 2 participants perceived less incapacity for the familiar IADL than at Visit $1(p=.01)$ and Visit $3(p=.01)$, with no differences between Visit 3 and baseline Visit $1(p=1.00)$. There was a moderate interaction between Group and Visit for familiar IADL, $F(2,43)=5.40, p<.01, \eta 2 \mathrm{p}=.21$. 
Analysis of the means and additional paired-sample $t$ tests for each group indicated the SenseCam group rates of incapacity for the FADL were lower at Visit 2 (M=11.82, $\mathrm{SD}=8.63)$, compared to Visit $1(\mathrm{M}=19.06, \mathrm{SD}=9.11), t(16)=4.91, p<.01, d=.816)$. Between Vist 2 and Visit 3, there was a marginal difference between Visit 3 (M=16.17, $\mathrm{SD}=8.90)$ and Visit $2,(t(14)=1.88, p=.08, d=.495)$, but there was no significant difference between Visit 3 and Visit 1 scores $(t(15)=.80, p=.44, d=.321)$. For $\mathrm{Memo}+$, the scores were significantly lower at Visit $2(\mathrm{M}=11.05, \mathrm{SD}=5.84)$ compared to Visit 1 $(\mathrm{M}=22 ., 86, \mathrm{SD}=16.14)$ with a strong effect size $(t(16)=4.96, p<.01, d=.973)$ but increased moderately again at Visit $3(\mathrm{M}=16.76, \mathrm{SD}=12.07), t(15)=-2.35, p=.03, d=.602)$, such that the differences between Visit 1 and 3 were only marginally significant $(t(14)=1.79, p=.09$, $d=.428$ ). In the Diary intervention, an opposite pattern was identified, with the rates of incapacity in familiar IADL increasing moderately after the intervention: Visit 2 $(\mathrm{M}=17.02, \mathrm{SD}=11.29), t(16)=-2.55, p=.02, d=.552$, compared to visit $1(\mathrm{M}=12.14$, $\mathrm{SD}=5.36)$ and remaining high at follow-up, Visit $3(\mathrm{M}=19.24, \mathrm{SD}=11.67), t(14)=-.94$, $p=.73, d=.193$, without any significant difference between Visits 1 and $3, \mathrm{t}(14)=-1.72$, $p=.11, d=.296$.

Concerning the advanced IADL, a similar pattern was found. No main effect of group was identified, $F(2,43)=.69, p=.507, \eta 2 p=.083$ but a moderate main effect of Visit was found $F(2,43)=11.74, p<.01, \eta^{2} \mathrm{p}=.22$, as well as a moderate interaction for Group $\mathrm{x}$ Visit, $F(2,43)=4.83, p<.01, \eta^{2}{ }^{2}=.19$. We again performed paired samples t-tests for each group across visits. The SenseCam group rated the items of the Advanced IADL lower at Visit $2(\mathrm{M}=21.82, \mathrm{SD}=13.10)$, compared to Visit 1 , and this was a strong difference between the two time-points $(\mathrm{M}=39.17, \mathrm{SD}=16.89), t(16)=4.77, p<.01, d=1.14)$ increasing again moderately at Visit 3, $(\mathrm{M}=31.41, \mathrm{SD}=17.85), t(14)=-2.45, p=.03, d=.595)$, but still remaining at a lower level than at Visit $1, t(14)=2.27, p=.04, d=.447$, with a moderate effect. In the Memo+ group, the scores for Advanced IADLs were also lower at Visit 2 $(\mathrm{M}=27.41, \mathrm{SD}=13.14)$, with a strong effect, compared to Visit $1(\mathrm{M}=40.38, \mathrm{SD}=13.86)$, $t(16)=4.67, p<.01, d=.96)$, but there were no significant differences between Visit 2 and Visit $3(\mathrm{M}=35.46, \mathrm{SD}=13.45), t(15)=-1.75, p=.09, d=.605$, and Visit 3 scores were similar to baseline scores, $t(15)=1.75, p=.10, d=.36$ for the Memo+ group. For the Diary group, no differences across Visit were identified for the Advanced IADL scores, neither between visit 1 and visit $2, t(16)=-1.427, p=.17, d=.15$ between visit 2 and $3, t(15)=-.896$, 
$p=.39, d=.043$ or between visit 1 and $3, t(15)=-1.718, p=.14, d=.24$ and if anything, scores go in the direction of worsening activities of daily living (Visit $1 \mathrm{M}=33.63$, $\mathrm{SD}=15.13$; Visit $2 \mathrm{M}=36.86, \mathrm{SD}=13.27$; Visit $3 \mathrm{M}=38.95, \mathrm{SD}=12.71$ ).

\section{Quality of Life}

We analysed the results of the World Health Organization Quality of Life - Older Adults module (WHOQOL-OLD, Vilar et al., 2010). A 3x3 Mixed ANOVA identified a moderate main effect of Group, $F(2,43)=5.30, p<.01, \eta^{2}=.20$. Fisher LSD post-hoc comparisons revealed that the SenseCam group perceived QoL higher than the control group - Diary (LSD, $p<.01$ ), with no differences detected between the SenseCam group and the Memo+ group (LSD, $p=.26$ ) neither between the Memo+ group and the Diary group (LSD, $p=.21$ ). A main weak to moderate effect of Visit was also found, $F(2,43)=$ 5.77, $p=.01, \eta^{2}=.12$, and LSD pairwise comparisons with Bonferroni adjustment identified increased perceived QoL in Visit 2 compared to both Visit $1(p=.01)$ and Visit 3 ( $p=.01)$, and no differences found between Visit 3 and Visit 1 ( $p=.52)$. However, no interaction effect was found for Group x Visit, $F(2,43)=1.69, p=.136, \eta 2 p=.052$, indicating that none of the interventions had a different effect on the QoL scores.

\section{DISCUSSION}

Whilst comparatively many studies have investigated the effect of cognitive rehabilitation programmes on cognitive function, there has been much less investigation of the impacts of such programmes on non-cognitive outcomes, and very little using standardized measures. Besides the lack of a good number of studies concerning other outcomes than cognition, the ones that do exist usually use measures not used frequently in clinical and research settings in the field of $\mathrm{AD}$. Additionally very few studies have tested, in the same study, cognitive, functional and emotional variables as outcomes of a cognitive intervention (Piras et al., 2011). The study presented here concerns the examination of three different cognitive programmes and their impact on wellbeing, and is part of a study where cognitive outcomes were also tested (Silva et al., submitted). The present study focussed, for the first time, on the effectiveness of a new wearable camera (SenseCam) to ameliorate mood, perceived quality of life and functional capacity in AD; this study 
was part of a larger research were a comprehensive neuropsychological assessment protocol was applied and the purpose here was to examine whether non-cognitive measures such as depressive symptoms, functional capacity and quality of life, were affected by the cognitive effects of these interventions.

Concerning the cognitive outcomes, we found that the SenseCam condition specifically improved autobiographical memory specificity (and this was even maintained at Visit 3) with moderate effects both for the intervention $(\eta 2 p=.23)$ and the interaction $(\eta 2 p=.43)$. This meant that, after post-hoc analysis, we could conclude that the interventions using SenseCam as an external memory aid contributed to generate more specific memories (we used the Autobiographical Memory Test, from Williams and Broadbent, 1986, where a set of fifteen cue-words, such as "surprised" "sad" "theatrical" were given for the participants to retrieve a personal event for each word and the memories retrieved could be either semantic associations, categorical memories, extended memories or specific memories - the last one being the highest level of memories' specificity (for a review on AM specificity see Williams et al., 2007). As previously stated the increase of memories specificity through training is known to improve mood and overall wellbeing (Raes et al., 2009). Besides these findings, the cognitive outcomes' analysis revealed that both SenseCam and Memo+ interventions improved general episodic and semantic memory, as well as some improvement in executive function. These effects were found both immediately after the end of the interventions and most of them remained at follow-up.

We were interested to examine whether the findings with the cognitive outcome generalized to measures of wellbeing. To the extent that depressive symptoms and quality of life are related to cognitive function, we expected to see similar patterns in our noncognitive measures. With the Geriatric Depression Scale, we found that both SenseCam and Memo+ programmes led to significant reductions in depressive symptoms, when tested immediately after the 11-week programme. The Diary control condition did not show such benefits. These reductions in depressive symptomology were not maintained at a longer term follow-up in either group, although there was a trend that depressive symptoms were lower than initially. This pattern is, at least partially, in accordance with our findings for the cognitive outcomes, as the SenseCam and the Memo+ conditions seem to be more effective than the Diary. 
Turning to the effect provided by SenseCam, we should expect that the fact that SenseCam is linked with the features of autobiographical memory, it should improve its specificity and consequently improve mood, as stated in the literature (Baquero and Martin, 2015; Gonçalves, Albuquerque and Paul, 2009), but this idea was as yet untested in Alzheimer's disease. The Memo+ condition also included some tasks designed to improve autobiographical memory specificity (based in Serrano et al., 2004), that again could explain the enhancement of wellbeing from the reduction of depressive symptoms. However, whereas the cognitive changes in autobiographical memory function were maintained at Visit 3 in the SenseCam group, we do not find such maintenance in the improved GDS scores. Paired samples t-tests revealed statistically significant differences between the assessment immediately after the end of the SenseCam intervention and at follow-up suggesting an increase of depressive symptoms at the final assessment, although not to the level of depressive symptomatology found at baseline or in the control group. The fact is that, whilst specificity remains high (according to our findings in the cognitive part of our research), there is significant a return of some depressive symptoms, meaning that we might question whether the mechanism is directly memory specificity, but some other generalised benefit or pleasure of using the device, for example.

For functional capacity, for the total score we found a similar pattern as for depressive symptomology, with the Memo+ and SenseCam groups showing clear benefits on activities of daily living. However, this pattern was not maintained at follow-up, returning to baseline level, with no significant differences between Visits 1 and 3 for SenseCam or for the Memo+ intervention). Here, we find a clearer pattern of short term gains in perceived functional capacity for the most effective cognitive interventions (SenseCam and Memo+) reinforced by the opposite pattern in the control condition; the Diary intervention participants rated their functional capacity progressively worse across the three time points. The patterns are broadly the same for the subscores, clear effects of both Memo+ and SenseCam, but there is a suggestion that for the advanced IADL these benefits may be retained at follow up in the SenseCam group.

As the advanced IADLs are known to be the first to be affected by the preclinical and mild stages of AD (Sikkes et al., 2011), and, as such, are those that need early neuropsychological interventions (Clare, Wilson, Carter, Hodges and Adams 2001; Clare et al., 2010), this short term effect of both SenseCam and Memo+ for advanced IADLs is 
of value for early intervention in AD (Silva et al., 2016). However, for future study, as we only accounted for perceived functional capacity, these findings should be correlated with more objective measures of functionality (direct observation, etc.) and also with third-person information.

Finally, for quality of life, despite of the presence of a main effect of group, with the SenseCam group having a better quality of life than the other groups, the absence of an interaction means it is difficult to attribute this difference to our intervention. Instead, with the significant main effect of Visit, we can interpret these data as showing that all groups benefit equally in terms of quality of life at the second time point, although we should also be cautious in our evaluation since the differences between groups on the baseline measure of quality of life did approach significance.

In summary, we find evidence of an impact of two of our memory programmes on measures of wellbeing, most notably depressive symptoms and perceived functional capacity. For no measure do we see an improvement in wellbeing for the Diary control group. We suggest that SenseCam yields modest improvements in mood in the longer term when compared with the Memo+-type intervention. This is an important adding to the existing literature, both towards the effects of external strategies in $\mathrm{AD}$ and specifically regarding the use of these new technological devices for both cognitive and wellbeing purposes. Because SenseCam is the most novel rehabilitation tool used here, we will focus on discussing that aspect of the data. In short, although SenseCam does not have a unique beneficial effect on wellbeing as we hypothesised, it is, at least, in comparison with the Memo+ programme, much less cognitively demanding and requires much less input from a third party or professional, and in that way, is a 'passive' memory aid.

For the depressive symptoms, despite the fact that there was an increase of depressive symptoms at follow up, that increase did not return to the baseline level in the SenseCam condition, which gives positive indicator for SenseCam usage not only for the improvement of cognitive function as measured by neuropsychological tests but also, possibly for its connection with the autobiographical memory neural circuits (Loveday and Conway, 2011; Silva et al., 2016). The fact that SenseCam reduces depressive 
symptoms is not surprising considering its effect on autobiographical memory specificity, and the knock-on effects on memory retrieveal. On the other hand, if we consider the experience of reviewing SenseCam images it is perhaps surprising. Before this study, it was possible to hypothesise that the patient being reminded of their forgetfulness or life situation would actually have a negative impact on mood, in line with a 'depressive realism' cognitive account of depressed mood (e.g. Moore and Fresco, 2012). If nothing else, this study shows that SenseCam review does not provoke negative evaluations of the loss of autobiographical memory, but as with early informal studies and case reports, suggests a beneficial action on mood.

This finding is in line with the research that suggests that wearable cameras are less demanding than other external aids that require training such as the daily diary control group used here, and are also less demanding and more motivating than paper and pencil cognitive exercises. So, one can conclude that compensatory interventions for mild AD that resort on these kind of devices have more global effects than traditional interventions that only target specific outcomes. Wearable cameras like SenseCam are then useful for improving well-being in mild AD. Our data add to other less well-powered studies which did not use standardised outcome measures, which have already mentioned that people, immediately after being tested with SenseCam, refer that the experience was pleasant, that they feel more confident and that it was helpful for their memory and for daily functioning (Berry et al., 2007; Loveday and Conway, 2011).

\section{Limitations of this study}

Our findings, on the whole, were not maintained at follow up, with a return of our measures to baseline levels for most groups and most assessments. Given the fact that, for six months, people were faced with no cognitive stimulation from any source, this is perhaps unsurprising and this contributed for the loss of the beneficial non-cognitive effects. What is interesting is that cognitive benefits were, however, maintained over six months, suggesting that it is not directly the changes in cognition which lead to improvement in wellbeing, but rather the use of the device itself. Another fact is that, for some of the measures (functional status, for instance), a less clear pattern was found (no group effects, but interactions), what might suggest that the measures used to test these variables are not adequate to test what they meant to test. 
In the case of the perceived functional status, we used an inventory no specifically constructed for Alzheimer's disease. Measures such as The Bayer Activities of Daily Living Scale (B-ADL) or the Alzheimer Co-operative Study - Activities of Daily Living (ADCS-ADL), are considered to be more useful tools than general ADL measures for the evaluation of treatment effects and the progression of the disease (Robert et al., 2010). Similarly, the quality of life instrument used in this study was also a general measure for geriatric population and not specific for $\mathrm{AD}$, when several measures such as the Alzheimer Disease Related Quality of Life (ADRQL), the Dementia Quality of Life Instrument (DQoL) or the Quality of Life - Alzheimer's Disease (QoL-AD) have been constructed considering the quality of life aspects more relevant for those patients.

Additional to these methodological limitations, third person evaluation would also have been useful, at least for the functional status ratings. The present study did not address the caregivers' perspective (see Loewenstein et al., 2004). Considering that, despite the patients tested being in the mild stages, they are possibly not completely aware of their real abilities to perform the activities of daily living. Thus, the perspective of a caregiver would be very helpful in this regard, although some confounding factors may arise and need to be taken in account, such as caregiver burden (Gitlin and Schulz, 2012).

\section{Conclusions}

Despite the methodological and sample size limitations of the present study, the findings are inspiring for future research. It is the first time SenseCam has been tested with standardized measures in comparison with other cognitive interventions in a group of mild AD patients, homogeneous in terms of the main sociodemographic variables and severity of the disease. In the Woodberry and colleagues study (2015) where SenseCam was tested with AD patients concerning memory for events depicted in the images, the subjective experience was examined by asking if they found the camera helpful, enjoyable to wear and whether seeing the images made them feel more confident. In the present study, we tried to examine a more global impact of this device in patients' daily life and we concluded that SenseCam is not only useful to stimulate cognitive function but also overall function (affective, functional) even in a neurodegenerative condition as AD. The apparent absence of long-term effects in the perceived wellbeing of this device might indicate the need to apply this strategy as a non-pharmacological intervention for AD for 
longer periods than what we did in this study (six weeks). Future research should address this time-related hypothesis as well as to ameliorate the outcome measures and increase the sample size. 


\section{Conflict of interest declaration}

The authors declare no conflict of interest

\section{Description of authors' roles}

Ana Rita Silva (ARS), Maria Salomé Pinho (MSP), and Luis Macedo (LM) formulated the research question and secured research funding for ARS's PhD position. The study was designed by ARS, MSP, LM and Chris Moulin (CM). The study was carried out by ARS with assistance from Horácio Firmino (HM) and Maria Salomé Caldeira (MSC). Data analysis was carried out by ARS with input from all other authors. ARS and CM drafted the article, with substantive comments and revisions provided by all authors.

\section{Acknowledgements}

This work was supported by funding from the Region of Bourgogne (FABER) and Fondation Médéric to Chris Moulin: "Conscience et Mémoire dans la maladie d"Alzheimer" and "Nouvelles technologies et Maladie d"Alzheimer: vers une utilisation optimale de l'autographer. This work was also supported by the Portuguese Foundation for Science and Technology, to Ana Rita Silva with a doctoral studentship for the project: "Memory stimulation in Mild Alzheimer's: the role of SenseCam in a comprehensive memory training program" [SFRH/BD/68816/2010]. 


\section{References}

Alves, L., Simões, M. R., and Martins, C. (2012). The estimation of premorbid intelligence levels among Portuguese speakers: The Irregular Word Reading Test (TeLPI). Archives of Clinical Neuropsychology, 27(1), 58-68. DOI:10.1093/arclin/acr103

Arkin, S. (2001). Alzheimer rehabilitation by students: Interventions and outcomes. Neuropsychological Rehabilitation, $\quad 11(3-4), \quad 273-317$. DOI:10.1080/09602010143000059

Bahar-Fuchs, A., Clare, L., and Woods, B. (2013). Cognitive training and cognitive rehabilitation for mild to moderate Alzheimer's disease and vascular dementia. Cochrane Database Systematic Reviews, 6, 1-100. DOI: 10.1002/14651858.CD003260.pub2

Baquero, M., and Martín, N. (2015). Depressive symptoms in neurodegenerative diseases. World Journal of Clinical Cases: WJCC, 3(8), 682-693. http://doi.org/10.12998/wjcc.v3.i8.682

Belleville, S. (2008) 'Cognitive training for persons with mild cognitive impairment', International Psychogeriatrics, 20(1), pp. 57-66. doi: 10.1017/S104161020700631X.

Berry, E., et al. (2007) The use of a wearable camera SenseCam as a pictorial diary to improve autobiographical memory in a patient with limbic encephalitis. Neuropsychological Rehabilitation; 17, 582-681. $\quad$ DOI: https://dx.doi.org/10.1080/09602010601029780

Bourgeois, M. (2007). Memory Books and Other Graphic Cuing Systems. New York: Health Professions Press, Paul H. Brookes Publishing.

Cammisuli, D.M. et al. (2016) Non-pharmacological interventions for people with Alzheimer's Disease: A critical review of the scientific literature from the last ten years, European Geriatric Medicine, Volume 7, Issue 1, 57 - 64

Cherry, K. E., Walvoord, A. A., and Hawley, K. S. (2010). Spaced retrieval enhances memory for a name-face-occupation association in older adults with probable Alzheimer's disease. The Journal of Genetic Psychology, 171, 168-181. DOI: $10.1080 / 00221320903548118$.

Choi, J., and Twamley, E. W. (2013). Cognitive rehabilitation therapies for Alzheimer's disease: A review of methods to improve treatment engagement and self- 
efficacy. Neuropsychology Review, 23(1), 48-62. http://doi.org/10.1007/s11065-0139227-4

Clare, L., et al. (2010). Goal-oriented cognitive rehabilitation for people with early-stage Alzheimer Disease: a single-blind randomized controlled trial of clinical efficacy. American Journal of Geriatric Psychiatry, 18, 928-939. DOI : 10.1097/JGP.0b013e3181d5792a

Clare, L., Linden, D. E., Woods, R. T., Whitaker, R., Evans, S. J., Parkinson, C. H., ... and Rugg, M. D. (2010). Goal-oriented cognitive rehabilitation for people with earlystage Alzheimer disease: a single-blind randomized controlled trial of clinical efficacy. The American journal of geriatric psychiatry, 18(10), 928-939

Clare, L., Wilson, B. A., Carter, G., Hodges, J. R., and Adams, M. (2001). Long-term maintenance of treatment gains following a cognitive rehabilitation intervention in early dementia of Alzheimer type: A single case study. Neuropsychological rehabilitation, 11(3-4), 477-494.

Crete-Nishihata, et al. (2012). Reconstructing the Past: Personal Memory Technologies Are Not Just Personal and Not Just for Memory. Human-Computer Interaction, 27(1-2), 92-123. doi: 10.1080/07370024.2012.656062

De Leo, G., Brivio, E., and Sautter, S. W. (2011). Supporting autobiographical memory in patients with Alzheimer's disease using smart phones, Applied Neuropsychology, 18(1), 69-76. DOI : 10.1080/09084282.2011.545730.

Dunn, J., and Clare, L. (2007). Learning face - name associations in early-stage dementia: Comparing the effects of errorless learning and effortful processing. Neuropsychological Rehabilitation, 17, 735 - $754 . \quad$ DOI: https://dx.doi.org/10.1080/09602010701218317

Fish, J., Wilson, B., and Manly, T. (2010). The assessment and rehabilitation of prospective memory problems in people with neurological disorders. A review. Neuropsychological Rehabilitation, 20, 161-178. DOI: https://dx.doi.org/10.1080\%2F09602010903126029

Folstein, M., Folstein, S., and McHugh, P. (1975) "Mini-mental state". A practical method for grading the cognitive state of patients for the clinician. Journal of Psychiatry Research, 12, 189-198.

Gitlin, L. N., and Schulz, R. (2012). Family caregiving of older adults. In T. R. Prohaska, L. A. Anderson, and R. H. Binstock (Eds.), Public Health for an Aging Society (pp. 181204). Baltimore, MD: Johns Hopkins University Press. 
Goncalves, D., Albuquerque, P., and Paul, C. (2009). Life review with older women: an intervention to reduce depression and improve autobiographical memory. Aging and Clinical Experimental Research, 21, 369-371.

Hampstead, B. M., Sathian, K., Phillips, P. A., Amaraneni, A., Delaune, W. R., and Stringer, A. Y. (2012). Mnemonic strategy training improves memory for object location associations in both healthy elderly and patients with amnestic mild cognitive impairment: a randomized, single-blind study. Neuropsychology,26(3), 385-399. http://doi.org/10.1037/a0027545

Haslam, C., Jetten, J., Haslam, S. A., Pugliese, C., and Tonks, J. (2011). 'I remember therefore I am, and I am therefore I remember': exploring the contributions of episodic and semantic self-knowledge to strength of identity. British Journal of Psychology, 102 (2), 184-203. DOI: 0.1348/000712610X508091

Hodges, S, et al. (2006). SenseCam: A retrospective memory aid. Ubiquitous Computing Proceedings, 4206, 177-193. DOI: http://dx.doi.org/10.1007/11853565_11

Huckans, M., Hutson, L., Twamley, E., Jak, A., Kaye, J., and Storzbach, D. (2013). Efficacy of cognitive rehabilitation therapies for mild cognitive impairment (MCI) in older adults: Working toward a theoretical model and evidence-based interventions. Neuropsychological Review, 23, 63-80. DOI: 10.1007/s11065-013-9230-9.

Jahn, H. (2013). Memory loss in Alzheimer's disease. Dialogues in Clinical Neuroscience, 15(4), 445-454.

Kapur, N., Glisky, E., and Wilson, B. (2004) Technological memory aids for people with memory deficits. Neuropsychological Rehabilitation. 14 (1/2), 41-60. DOI: http://dx.doi.org/10.1080/09602010343000138

Lee, M., and Dey, A. (2008). Lifelogging memory applicance for people with episodic memory impairment. Conference on Ubiquitous Computing. Ubicomp '08 Proceedings. DOI: http://dx.doi.org/10.1145/1409635.1409643

Lee, S., et al. (2009) Effects of spaced retrieval training on cognitive function in Alzheimer's disease (AD) patients. Archives of Gerontology and Geriatrics, 49, 289-293. DOI: 10.1016/j.archger.2008.10.005.

Loewenstein, D. A., Acevedo, A., Czaja, S. J., and Duara, R. (2004). Cognitive rehabilitation of mildly impaired Alzheimer's disease patients on cholinesterase inhibitors. American Journal of Geriatric Psychiatry, 12, 395-402. DOI: https://dx.doi.org/10.1176/appi.ajgp.12.4.395 
Loveday, C., and Conway, M. (2011). Using SenseCam with an amnesic patient: Accessing inaccessible everyday memories, Memory, 19, 697-704. DOI: 10.1080/09658211.2011.610803.

Martin, M., Clare, L., Altgassen, A., Cameron, M., and Zehnder, F. (2011). Cognition-based interventions for healthy older people and people with mild cognitive impairment. Cochrane Database of Systematic Reviews 2011, Issue 1. 1-32. Art. No.: CD006220. DOI: 10.1002/14651858.CD006220.pub2

McKhann, G. M., et al. (2011). The diagnosis of dementia due to Alzheimer's disease: Recommendations from the National Institute on Aging-Alzheimer's Association workgroups on diagnostic guidelines for Alzheimer's disease. Alzheimer's and Dementia: The Journal of the Alzheimer's Association,7(3), 263-269. http://doi.org/10.1016/j.jalz.2011.03.005

Mioshi, E., Dawson, K., Mitchell, J., Arnold, R., Hodges, J.R. (2006) The Addenbrooke's Cognitive Examination Revised (ACE-R): a brief cognitive test battery for dementia screening. International Journal of Geriatric Psychiatry 21, 1078-1085. DOI: https://dx.doi.org/10.1002/gps.1610

Moore, M. T., and Fresco, D. M. (2012). Depressive realism: a meta-analytic review. Clinical psychology review, 32(6), 496-509.

Morris, C. et al. (1993). The consortium to establish a registry for Alzheimer's disease. Part IV. Rates of cognitive change in the longitudinal assessment of probable Alzheimer's disease. Neurolog, 43, 2457-2465.

Olazarán, J. et al., (2010). Nonpharmacological Therapies in Alzheimer's Disease: A Systematic Review of Efficacy. Dementia and other Geriatric Cognitive Disorders, 30, 161-178.

Piras, F., Borella, E., Incoccia, C., and Carlesimo, G.A. (2011). Evidence-based practice recommendations for memory rehabilitation. European Journal of Physical and Rehabilitation Medicine, 47, 149-175. . ISSN: 1973-9087.

Raes, F., Williams, J. M. G., and Hermans, D. (2009). Reducing cognitive vulnerability to depression: A preliminary investigation of MEmory Specificity Training (MEST) in inpatients with depressive symptomatology. Journal of behavior therapy and experimental psychiatry, 40(1), 24-38. http://dx.doi.org/10.1016/j.jbtep.2008.03.001

Rathbone, C. J., Holmes, E. A., Murphy, S. E., and Ellis, J. A. (2015). Autobiographical memory and well-being in aging: The central role of semantic self- 
images. Consciousness and cognition, 33, 422-431. http://dx.doi.org/10.1016/j.concog.2015.02.017

Robert, P., Ferris, S., Gauthier, S., Ihl, R., Winblad, B., and Tennigkeit, F. (2010). Review of Alzheimer's disease scales: is there a need for a new multi-domain scale for therapy evaluation in medical practice? Alzheimer's Research \& Therapy, 2(4), 24. http://doi.org/10.1186/alzrt48

Salomone, S., Caraci, F., Leggio, G. M., Fedotova, J., and Drago, F. (2012). New pharmacological strategies for treatment of Alzheimer's disease: focus on disease modifying drugs. British Journal of Clinical Pharmacology, 73(4), 504-517. http://doi.org/10.1111/j.1365-2125.2011.04134.x

Seidl U., Lueken U., Thomann P. A., Geider J., and Schröder J. (2011). Autobiographical memory deficits in Alzheimer's disease. Journal of Alzheimer Disease. 27, 567-574. http://doi.org/10.3233/JAD-2011-110014

Silva, A., Pinho, S., Macedo, L. and Moulin, C (2016). A critical review of the effects of wearable cameras on memory. Neuropsychological Rehabilitation, 6 : 1-25. DOI : https://dx.doi.org/10.1080/09602011.2015.1128450

Silva, A., Pinho, S., Macedo, L. and Moulin, C (submitted) Testing the promise of new technology: The cognitive effects of wearable cameras in Mild Alzheimer disease.

Silva, A.R, Pinho, S., Macedo, L., and Moulin C.J. (2013) Immediate benefits of SenseCam review on neuropsychological test performance, American Journal of Preventive Medicine, 44(3):302-207. DOI: 10.1016/j.amepre.2012.11.005.

Thivierge, S., Simard, M., Jean, L., and Grandmaison, E. (2008) Errorless learning and spaced retrieval techniques to relearn instrumental activities of daily living in mild Alzheimer's disease: a case report study. Neuropsychiatric Disease and Treatment, 4(5), 987-999.

Williams, J. M. G., and Broadbent, K. (1986). Autobiographical memory in suicide attempters. Journal of Abnormal Psychology, 95, 144 -149.

Williams, J. M. G., Barnhofer, T., Crane, C., Herman, D., Raes, F., Watkins, E., and Dalgleish, T. (2007). Autobiographical memory specificity and emotional disorder. Psychological bulletin, 133(1), 122-149.

Woodberry, E., Browne, G., Hodges, S., Watson, P., Kapur, N., and Woodberry, K. (2015). The use of a wearable camera improves autobiographical memory in patients with Alzheimer Disease. Memory, 23, 340- 349. DOI: 10.1080/09658211.2014.886703. 
Yesavage , J. et al., (1983). Development and validation of a geriatric depression screening scale: a preliminary report. Journal of Psychiatry Research, 17, 37-42.

Zec, R. and Burkett, N. (2008). Non-pharmacological and pharmocological treatment of the cognitive and behavioural symptoms of Alzheimer's disease. Neurorehabilitation, $23,425-438$. 
Tables

Table 1. Demographic characteristics of the three cognitive intervention groups, at visit $1(\mathrm{~N}=\mathbf{5 1})$

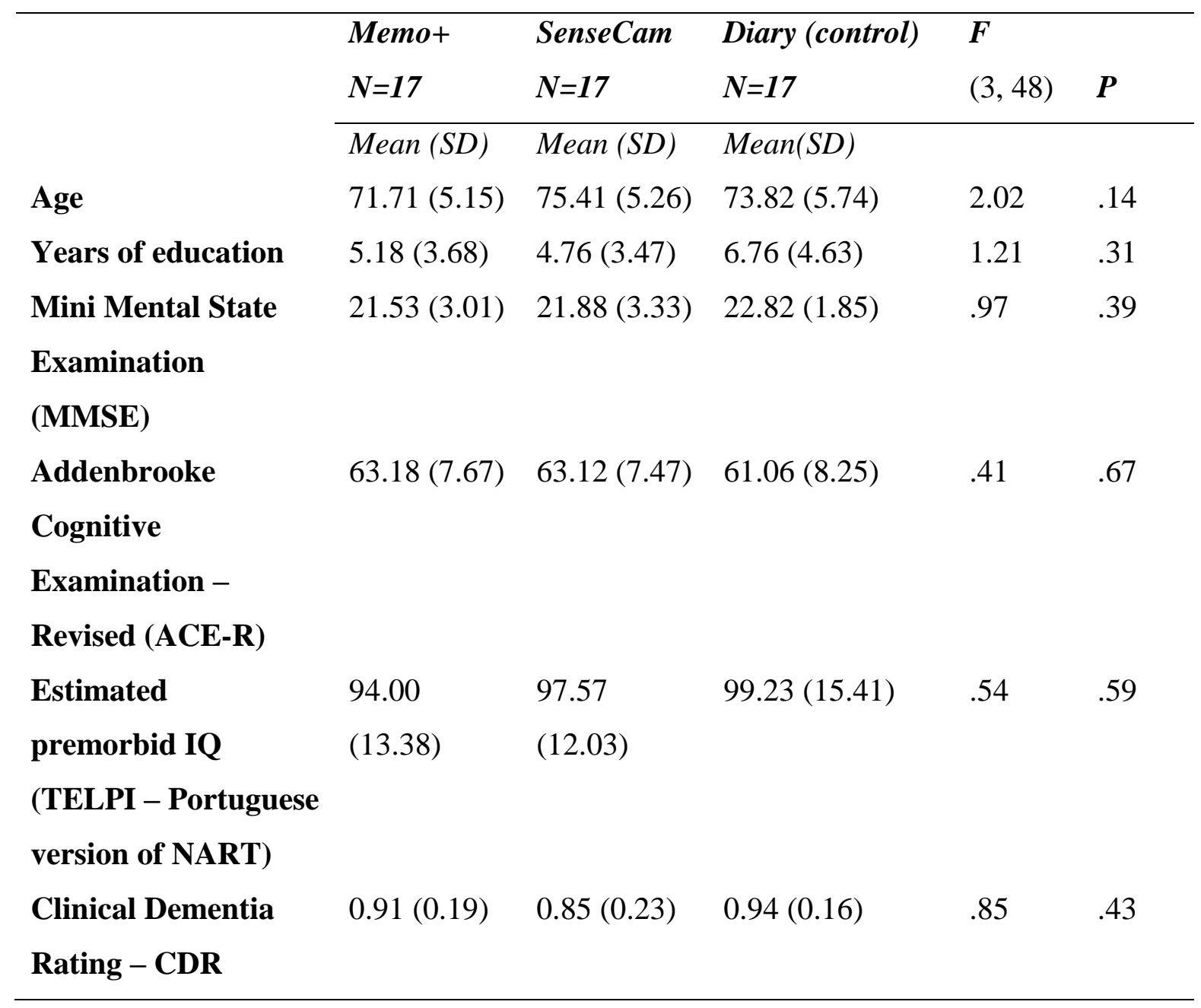


Table 2. Means, standard deviation and $p$ values for group differences in the non-cognitive measures assessed

\begin{tabular}{|c|c|c|c|c|c|c|c|c|c|c|}
\hline \multirow{4}{*}{$\begin{array}{l}\text { Instrument } \\
\text { Depressive Symptoms }\end{array}$} & \multicolumn{3}{|c|}{ SenseCam } & \multicolumn{3}{|c|}{ Memo+ } & \multicolumn{3}{|c|}{ Diary (control) } & \multirow{2}{*}{$\begin{array}{l}\text { Main effect } \\
\quad \text { of } \\
\text { intervention }\end{array}$} \\
\hline & 1 & 2 & 3 & 1 & 2 & 3 & 1 & 2 & 3 & \\
\hline & $\mathrm{N}=17$ & $\mathrm{~N}=17$ & $\mathrm{~N}=15$ & $\mathrm{~N}=17$ & $\mathrm{~N}=17$ & $\mathrm{~N}=16$ & $\mathrm{~N}=17$ & $\mathrm{~N}=17$ & $\mathrm{~N}=16$ & \\
\hline & & & & & & & & & & \\
\hline $\operatorname{GDS}(0-30)^{\mathrm{a}}$ & $\begin{array}{l}12.64 \\
(6.25)\end{array}$ & $\begin{array}{l}6.79 * \\
(3.66)\end{array}$ & $\begin{array}{l}7.57 \\
(4.03)\end{array}$ & $\begin{array}{l}11.44 \\
(4.62)\end{array}$ & $\begin{array}{l}10.31 \\
(5.12)\end{array}$ & $\begin{array}{l}11.06 \\
(4.50)\end{array}$ & $\begin{array}{l}13.00 \\
(5.29)\end{array}$ & $\begin{array}{l}13.40 \\
(5.22)\end{array}$ & $\begin{array}{l}14.60 \\
(5.12)\end{array}$ & $\begin{array}{l}3.53 \\
\mathrm{p}=.03\end{array}$ \\
\hline Functional capacity & & & & & & & & & & \\
\hline $\begin{array}{l}\text { IAFAI global (0- } \\
100)^{\mathrm{b}}\end{array}$ & $\begin{array}{l}19.72 \\
(7.74)\end{array}$ & $\begin{array}{l}11.58 \\
(8.18)\end{array}$ & $\begin{array}{l}16.92 \\
(8.36)\end{array}$ & $\begin{array}{l}21.42 \\
(11.54)\end{array}$ & $\begin{array}{l}14.06 \\
(7.54)\end{array}$ & $\begin{array}{l}18.26 \\
(9.64)\end{array}$ & $\begin{array}{l}16.07 \\
(5.96)\end{array}$ & $\begin{array}{l}18.40 \\
(7.42)\end{array}$ & $\begin{array}{l}19.58 \\
(7.32)\end{array}$ & n.s \\
\hline IAFAI basic & $\begin{array}{l}6.93 \\
(7.22)\end{array}$ & $\begin{array}{l}3.98 \\
(7.08)\end{array}$ & $\begin{array}{l}5.93 \\
(7.42)\end{array}$ & $\begin{array}{l}5.72 \\
(7.59)\end{array}$ & $\begin{array}{l}3.60 \\
(4.43)\end{array}$ & $\begin{array}{l}5.33 \\
(7.06)\end{array}$ & $\begin{array}{l}4.62 \\
(3.66)\end{array}$ & $\begin{array}{l}5.64 \\
(4.26)\end{array}$ & $\begin{array}{l}4.86 \\
(4.22)\end{array}$ & n.s. \\
\hline $\begin{array}{l}\text { IAFAI instrumental } \\
\text { familiar }\end{array}$ & $\begin{array}{l}18.72 \\
(9.96)\end{array}$ & $\begin{array}{l}11.11 \\
(8.26)\end{array}$ & $\begin{array}{l}16.17 \\
(8.90)\end{array}$ & $\begin{array}{l}22.56 \\
(16.62)\end{array}$ & $\begin{array}{l}11.24 \\
(5.96)\end{array}$ & $\begin{array}{l}16.76 \\
(12.07)\end{array}$ & $\begin{array}{l}12.14 \\
(5.36)\end{array}$ & $\begin{array}{l}17.02 \\
(11.28)\end{array}$ & $\begin{array}{l}19.24 \\
(11.67)\end{array}$ & n.s. \\
\hline $\begin{array}{l}\text { IAFAI instrumental } \\
\text { advanced }\end{array}$ & $\begin{array}{l}37.85 \\
(15.02)\end{array}$ & $\begin{array}{l}23.62 \\
(13.81)\end{array}$ & $\begin{array}{l}31.41 \\
(17.85)\end{array}$ & $\begin{array}{l}40.51 \\
(14.30)\end{array}$ & $\begin{array}{l}28.58 \\
(12.62)\end{array}$ & $\begin{array}{l}35.46 \\
(13.45)\end{array}$ & $\begin{array}{l}34.04 \\
(15.91)\end{array}$ & $\begin{array}{l}36.32 \\
(14.06)\end{array}$ & $\begin{array}{l}38.95 \\
(12.71)\end{array}$ & n.s. \\
\hline Quality of Life & & & & & & & & & & \\
\hline $\begin{array}{l}\text { WHOQOL-OLD } \\
(0-)^{c}\end{array}$ & $\begin{array}{l}109.33 \\
(15.64)\end{array}$ & $\begin{array}{l}116.47 * \\
(12.71)\end{array}$ & $\begin{array}{l}110,00 \\
(16.73)\end{array}$ & $\begin{array}{l}103.75 \\
(12.86)\end{array}$ & $\begin{array}{l}107.19 \\
(11.26)\end{array}$ & $\begin{array}{l}103.38 \\
(10.28)\end{array}$ & $\begin{array}{l}100.27 \\
(10.34)\end{array}$ & $\begin{array}{l}99.20 \\
(9.66)\end{array}$ & $\begin{array}{l}91.27 \\
(24.67)\end{array}$ & $\begin{array}{l}5.31 \\
\mathrm{p}<.01\end{array}$ \\
\hline
\end{tabular}


${ }^{a}$ For this measure a higher score indicates higher levels of depressive symptomatology. Note that patients whose score was above 20 at baseline (meaning severe depressive symptoms) were excluded from the study.

${ }^{\mathrm{b}}$ For this measure a higher score indicates the perception of higher levels of functional incapacity.

${ }^{c}$ For this measure, a higher score would suggest better perceived quality of life.

*Indicates the presence of a significant statistical difference with the control group. 
EFFECTS OF SENSECAM ON IMPROVING WELL-BEING IN PATIENTS WITH MILD AD

\section{Graphs}

Graph 1. Mean score for the Geriatric Depressive Scale (GDS) for the three cognitive interventions at the three moments of assessment

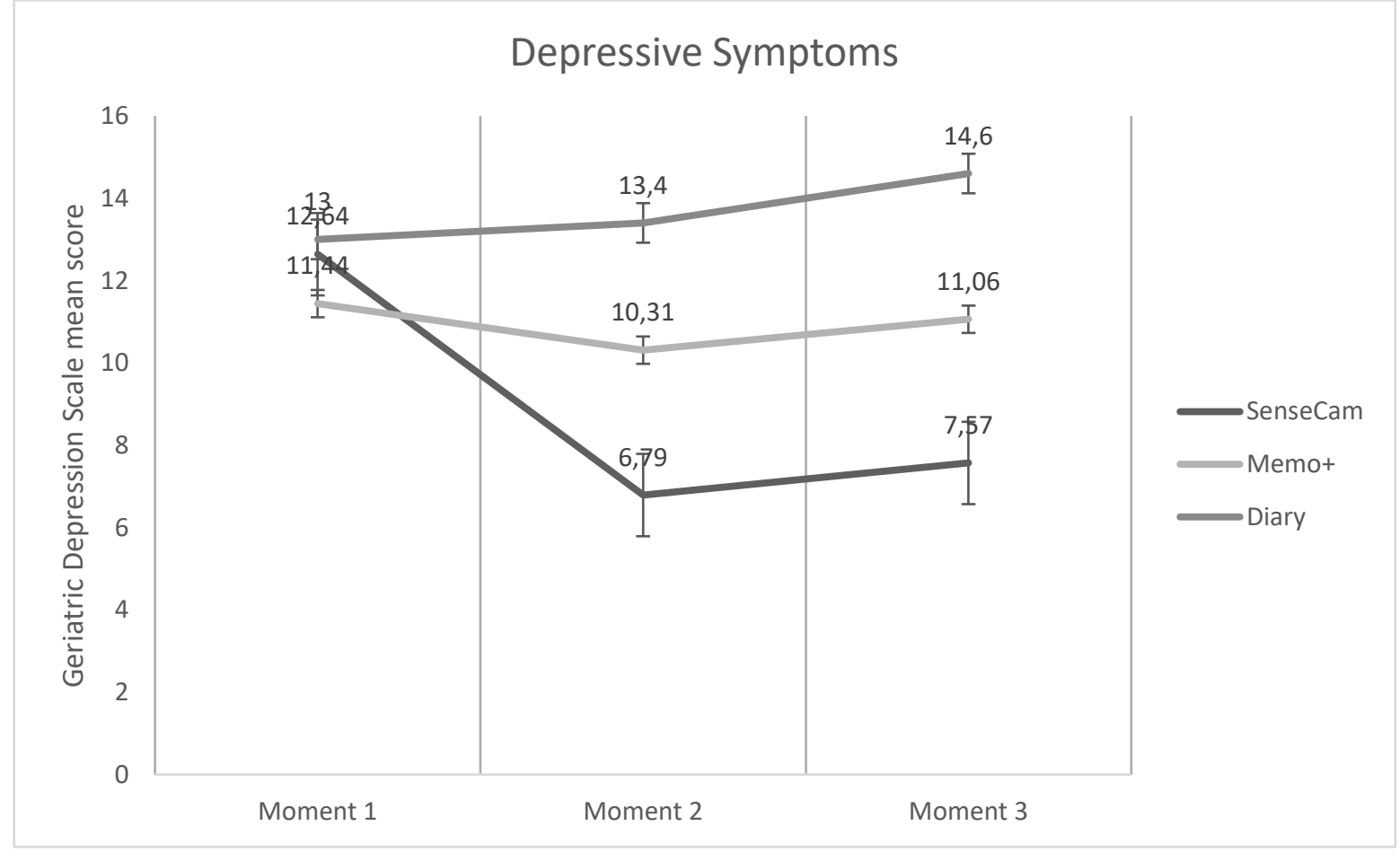

